\title{
Substantial Effects of Luseogliflozin Revealed by Analyzing Responses to Postprandial Hyperglycemia: Post Hoc Subanalyses of a Randomized Controlled Study
}

Yoshishige Samukawa · Hirohisa Omiya · Hirotaka Watase •

Kazunari Nozaki · Soichi Sakai · Rimei Nishimura

Received: April 12, 2016/ Published online: June 2, 2016

(C) The Author(s) 2016. This article is published with open access at Springerlink.com

\begin{abstract}
Introduction: In our previous study investigating effects of luseogliflozin, a sodium-glucose cotransporter 2 inhibitor, on 24-h glycemic variability by continuous glucose monitoring (CGM), luseogliflozin elicited parallel downward shifts in fasting and postprandial glucose levels. However, further review of individual patients' data revealed that postprandial hyperglycemia was not reduced in some patients, while preprandial glucose was ameliorated in most patients. Therefore, we
\end{abstract}

Enhanced content To view enhanced content for this article go to http://www.medengine.com/Redeem/54D4 F0602980E586.

Electronic supplementary material The online version of this article (doi:10.1007/s12325-016-0350-5) contains supplementary material, which is available to authorized users.

Y. Samukawa $(\bowtie) \cdot H$. Omiya · H. Watase .

K. Nozaki · S. Sakai

Taisho Pharmaceutical Co., Ltd., 3-24-1 Takada,

Toshima-ku, Tokyo 170-8633, Japan

e-mail: y-samukawa@so.taisho.co.jp

R. Nishimura

Division of Diabetes, Metabolism and

Endocrinology, Department of Internal Medicine,

Jikei University School of Medicine, 3-19-18

Nishi-shimbashi, Minato-ku, Tokyo 105-0003, Japan divided patients into two groups according to their postprandial glucose responses and conducted a post hoc subanalyses to elucidate which factors contributed to the differential effects of luseogliflozin.

Methods: Thirty-four Japanese type 2 diabetic patients in our previous randomized, double-blind, placebo-controlled, crossover study with 7-day luseogliflozin administration were divided into postprandial glucose responders (PGR, $n=23$, ameliorated peak glucose) and postprandial glucose non-responders (PGNR; $n=11$, non-ameliorated peak glucose). Baseline characteristics, variations in CGM-measured 24-h glucose levels, and other pharmacodynamic variabilities were compared. Results: Baseline characteristics did not differ significantly between groups. Placebo-subtracted peak glucose was significantly lowered in PGR and significantly increased in PGNR (-43.8 and $17.9 \mathrm{mg} / \mathrm{dL}$; both $p<0.05)$. Luseogliflozin significantly lowered "lowest glucose" (defined as the lowest level measured throughout a $24-\mathrm{h}$ period) similarly in PGR and PGNR (-19.2 and $-24.0 \mathrm{mg} / \mathrm{dL}$; both $p<0.05)$, significantly reduced the mean 
amplitude of glucose excursions in PGR $(-15.50 \mathrm{mg} / \mathrm{dL} ; \quad p<0.05)$, and increased the area under the curve for plasma glucagon over $24 \mathrm{~h}$ in PGNR (median difference vs. placebo: $240 \mathrm{pg} / \mathrm{mL} \mathrm{h} ; p<0.05)$. Luseogliflozin increased urinary glucose excretion (UGE) and decreased serum insulin by similar magnitudes in both groups.

Conclusions: Luseogliflozin diminished glucose fluctuations in most patients by lowering peak glucose to a greater extent than lowest glucose. Luseogliflozin may also lower lowest glucose in patients whose peak glucose was not ameliorated despite increasing UGE. The glucagon increase in PGNR might explain its hypoglycemic effect on postprandial glucose. Funding: Taisho Pharmaceutical Co., Ltd, Tokyo, Japan.

Trial Registration: JapicCTI-142548.

Keywords: Continuous glucose monitoring; Diabetes; Japan; Luseogliflozin; Post hoc subanalysis; Postprandial hyperglycemia; Randomized controlled trial; Sodium-glucose cotransporter 2 inhibitor; Type 2 diabetes mellitus

\section{INTRODUCTION}

The updated American Diabetes Association (ADA)/European Association for the Study of Diabetes (EASD) position statement [1] evaluated recent data and developed recommendations, including the advantages and disadvantages, for anti-hyperglycemic agents for the treatment of type 2 diabetes mellitus (T2DM). The position statement places emphasis on a patient-centered approach, in which patient preference, cost, potential side effects, effects on body weight, and risk of hypoglycemia associated with each drug class should be considered when selecting a treatment approach.

Sodium-glucose cotransporter 2 (SGLT2) inhibitors are newly approved drugs that correct hyperglycemia without increasing the risk of hypoglycemia or body weight gain [2-6], and have been included as a treatment option for combination therapy of T2DM in the ADA/ EASD position statement [1]. To date, however, few studies have examined the effect of SGLT2 inhibitors on 24-h glycemic variability including nocturnal glycemia and the risk of asymptomatic hypoglycemia. Moreover, no studies have described the effects of SGLT2 inhibitors on the changes in serum insulin or plasma glucagon concentrations throughout the day; these data are important in terms of understanding the effects of these drugs on pancreatic $\alpha$ and $\beta$ cells $[7,8]$.

Luseogliflozin is a highly selective SGLT2 inhibitor that was recently approved and launched in Japan for the treatment of T2DM [5]. In a recent phase III study, luseogliflozin monotherapy significantly reduced circulating glycated hemoglobin (HbA1c), fasting plasma glucose (FPG), postprandial plasma glucose levels, body weight, and abdominal circumference over 24 weeks of treatment in Japanese patients with T2DM [9]. Luseogliflozin was well tolerated, with most adverse events (AEs), including hypoglycemia, rated as mild in severity.

Current treatments for T2DM aim to reduce the HbA1c concentration. However, while HbA1c reflects glucose concentrations over the preceding 1-3 months, it does not reflect daily glucose variability, such as early morning hypoglycemia or postprandial hyperglycemia.

We recently conducted a randomized, double-blind, placebo-controlled, crossover study to determine the effects of luseogliflozin on 24-h glucose levels assessed by continuous 
glucose monitoring (CGM) in Japanese patients with T2DM. The results of this study revealed that the glucose level was consistently lowered throughout the day in the luseogliflozin group (i.e., luseogliflozin produced a parallel downward shift in both fasting and postprandial glucose) $[10,11]$. Similar glycemic-lowering effects on 24-h glucose levels were also reported in a clinical study of empagliflozin, another SGLT2 inhibitor [12].

However, when we reviewed in detail the changes in 24-h glucose levels in the individual patients' data from our study [10], we found that postprandial hyperglycemia was not reduced in some patients, even though fasting glucose was ameliorated after luseogliflozin administration in almost all patients. Therefore, we divided the patients into two groups according to their responses to peak glucose, and performed post hoc subanalyses to elucidate the factors associated with the differential effects of luseogliflozin in these patients.

\section{METHODS}

\section{Study Design and Participants}

The present subanalyses are based on a previous randomized, double-blind, placebo-controlled, crossover study on the effects of luseogliflozin on 24-h glucose levels in Japanese patients with T2DM [10]. All procedures followed were in accordance with the ethical standards of the responsible committee on human experimentation (institutional and national) and with the Helsinki Declaration of 1964, as revised in 2013. Informed consent was obtained from all patients for being included in the study. The study protocol was approved by the Institutional Review Board of each study institute. This study was registered with the Japan Pharmaceutical Information Center (identifier: JapicCTI-142548).

Japanese patients with T2DM inadequately controlled with diet and exercise (HbA1c 7.0-10.0\%) were randomized into two groups in which patients received luseogliflozin $2.5 \mathrm{mg}$ followed by placebo for 7 days each, or vice versa. Please see Appendix S1 in the supplementary material for a list of the major exclusion criteria. Each treatment period was separated by a washout period of 7-14 days. Patients were hospitalized on day 7 and consumed a standardized meal $(536 \mathrm{kcal}$, with approximately $20 \%$ protein, $25 \%$ fat, and $55 \%$ carbohydrate) at breakfast, lunch, and dinner. CGM was performed on day 7 under hospitalization. Glucagon was measured by an established radioimmunoassay method. All laboratory tests were analyzed at a central laboratory, LSI Medience Corporation (Tokyo, Japan).

The study drug allocation manager randomly allocated the study drugs to both study groups, prepared a randomization schedule that matched the study drug number to the study groups, and sealed and kept the schedule until the code was broken. The investigators allocated a serial drug allocation number assigned by the study drug allocation manager to each eligible patient. The study drug and placebo were indistinguishable by appearance.

For these subanalyses, 34 patients who were enrolled to our previous study [10] were divided into two groups. Postprandial glucose responders (PGR, $n=23$ ) were defined as patients with peak glucose levels ameliorated by luseogliflozin treatment compared with placebo ( $>0 \mathrm{mg} / \mathrm{dL}$ after rounding to one decimal place). Postprandial glucose non-responders (PGNR, $n=11$ ) were defined 
as patients with peak glucose levels not ameliorated by luseogliflozin treatment compared with placebo $(\leq 0 \mathrm{mg} / \mathrm{dL}$ after rounding to one decimal place).

\section{Statistical Analysis}

We investigated and compared the baseline values, the effect on 24-h glucose variability, and other pharmacodynamic variability changes in the PGR and PGNR groups. Baseline characteristics are presented as the mean \pm standard deviation (SD). Regarding pharmacodynamic and glucose variables, normally distributed variables are presented as the least-squares mean (LSM) and two-sided 95\% confidence interval. The differences between the two treatments were analyzed using a mixed-effects model, which included treatment, sequence, and period as fixed effects and patients as a random effect. Non-normally distributed variables are presented as the median and interquartile range, and differences between the two treatments were determined using paired Wilcoxon tests. To evaluate the difference between PGR and PGNR groups in terms of the difference between luseogliflozin and placebo in each group, a two-sample $t$ test was used to analyze normally distributed variables. The Wilcoxon rank sum test was used to analyze non-normally distributed variables. All statistical analyses were performed using SAS software version 9.2 (SAS Institute Japan, Tokyo, Japan).

\section{RESULTS}

\section{Patient Baseline Characteristics}

Baseline demographic and clinical characteristics of the study patients are shown in Table 1 . There were no significant differences between the PGR and PGNR for HbA1c (mean \pm SD; $7.81 \% \pm$ $0.80 \%$ vs $7.70 \% \pm 0.67 \%)$, FPG $(160.3 \pm 28.0$ vs $154.7 \pm 20.3 \mathrm{mg} / \mathrm{dL}$ ), glycated albumin (GA; $21.67 \% \pm 4.08 \%$ vs $22.19 \% \pm 2.72 \%$ ), fasting serum insulin $(8.756 \pm 4.518$ vs $6.972 \pm 4.393$ $\mu \mathrm{U} / \mathrm{mL})$, fasting plasma glucagon $(83.0 \pm 28.7$ vs $92.3 \pm 32.3 \mathrm{pg} / \mathrm{mL})$, or estimated glomerular filtration rate (eGFR; $86.5 \pm 13.9$ vs $82.4 \pm$ $18.0 \mathrm{~mL} / \mathrm{min} / 1.73 \mathrm{~m}^{2}$ ).

\section{Pharmacodynamics}

The variations in 24-h glucose levels measured by CGM after 7 days of treatment with luseogliflozin and placebo in each group are shown in Fig. 1, and the indices derived from the CGM data are presented in Table 2.

Because patients were divided according to the responses in postprandial glucose concentrations, the placebo-subtracted peak glucose level throughout the day was significantly decreased in the PGR $(p<0.001$, Table 2) and was significantly increased in the PGNR ( $p=0.004$, Table 2). Consistent with this, the placebo-subtracted peak glucose levels after each meal were also significantly decreased in the PGR (all $p<0.001$, Table 2). Such changes were not observed in the PGNR.

Luseogliflozin significantly lowered the lowest glucose throughout the day, as well preprandial (before each meal) and midnight (3 h after sleep) glucose compared with placebo in the PGR and PGNR (all $p<0.05$, Table 2). Furthermore, daily glucose fluctuations for the mean amplitudes of glucose excursions (MAGE) and for SD over $24 \mathrm{~h}$ were significantly decreased in the PGR $(p=0.009$ and $p<0.001$, respectively, Table 2). By contrast, MAGE and SD over $24 \mathrm{~h}$ increased significantly in the PGNR ( $p=0.002$ and $p<0.001$, respectively).

The urinary glucose excretion (UGE) rate on day 7 is shown in Fig. 2a, and cumulative UGE 
Table 1 Patient characteristics at baseline

\begin{tabular}{|c|c|c|c|}
\hline & PGR & PGNR & $p$ value $^{\mathrm{a}}$ \\
\hline$n$ & 23 & 11 & - \\
\hline Male & $15(65.2)$ & $9(81.8)$ & - \\
\hline Age (years) & $60.0 \pm 8.3$ & $64.0 \pm 8.8$ & 0.202 \\
\hline Body weight (kg) & $66.07 \pm 14.83$ & $64.58 \pm 11.54$ & 0.772 \\
\hline BMI $\left(\mathrm{kg} / \mathrm{m}^{2}\right)$ & $25.03 \pm 3.90$ & $23.84 \pm 3.01$ & 0.380 \\
\hline Duration of diabetes (years) & $7.9 \pm 4.1$ & $5.8 \pm 4.0$ & 0.184 \\
\hline HbAlc (\%) & $7.81 \pm 0.80$ & $7.70 \pm 0.67$ & 0.689 \\
\hline $\mathrm{FPG}(\mathrm{mg} / \mathrm{dL})$ & $160.3 \pm 28.0$ & $154.7 \pm 20.3$ & 0.561 \\
\hline Glycated albumin (\%) & $21.67 \pm 4.08$ & $22.19 \pm 2.72$ & 0.704 \\
\hline Fasting serum insulin $(\mu \mathrm{U} / \mathrm{mL})$ & $8.756 \pm 4.518$ & $6.972 \pm 4.393$ & 0.285 \\
\hline Fasting plasma glucagon $(\mathrm{pg} / \mathrm{mL})$ & $83.0 \pm 28.7$ & $92.3 \pm 32.3$ & 0.405 \\
\hline $\mathrm{eGFR}\left(\mathrm{mL} / \mathrm{min} / 1.73 \mathrm{~m}^{2}\right)$ & $86.5 \pm 13.9$ & $82.4 \pm 18.0$ & 0.476 \\
\hline
\end{tabular}

Data are the mean \pm standard deviation or $n(\%)$

Glucose: $1 \mathrm{mg} / \mathrm{dL}=0.0556 \mathrm{mmol} / \mathrm{L}$

Insulin: $1 \mu \mathrm{U} / \mathrm{mL}=6.945 \mathrm{pmol} / \mathrm{L}$

Glucagon: $1 \mathrm{pg} / \mathrm{mL}=1 \mathrm{ng} / \mathrm{L}$

$B M I$ body mass index, $e G F R$ estimated glomerular filtration rate, FPG fasting plasma glucose, HbAlc glycated hemoglobin, $P G R$ postprandial glucose responders, $P G N R$ postprandial glucose non-responders

a Two-sample $t$ test

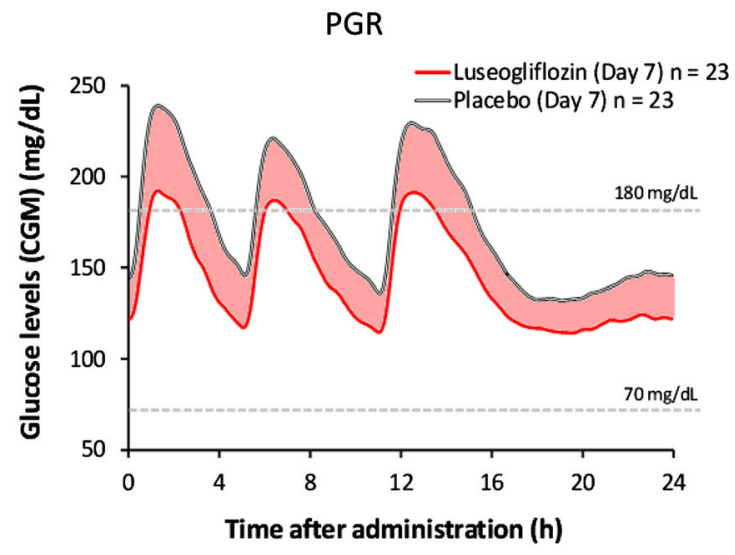

Fig. 1 Variations in 24-h glucose levels. 24-h glucose level assessed by continuous glucose monitoring $(1 \mathrm{mg} /$ $\mathrm{dL}=0.0556 \mathrm{mmol} / \mathrm{L})$. Values are presented as the mean (error bars were omitted for clarity). The red area indicates a reduction in glucose levels by luseogliflozin vs placebo.

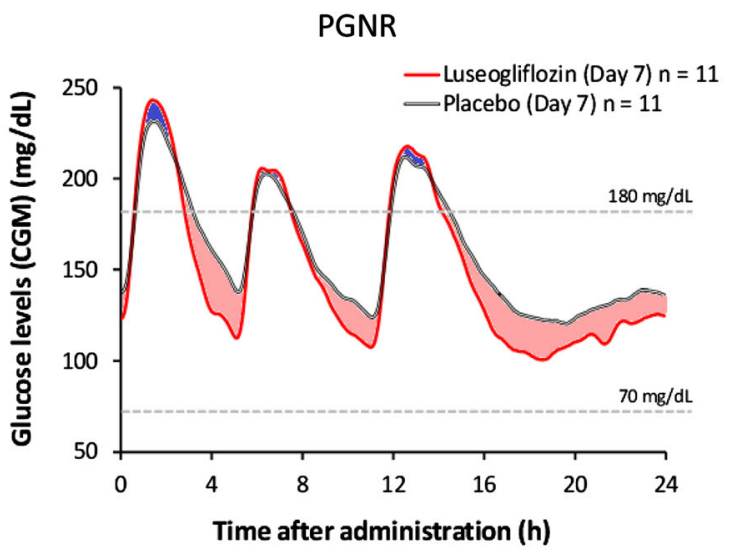

The blue area indicates an increase in glucose levels by luseogliflozin vs placebo. CGM continuous glucose monitoring, $P G R$ postprandial glucose responders, $P G N R$ postprandial glucose non-responders 


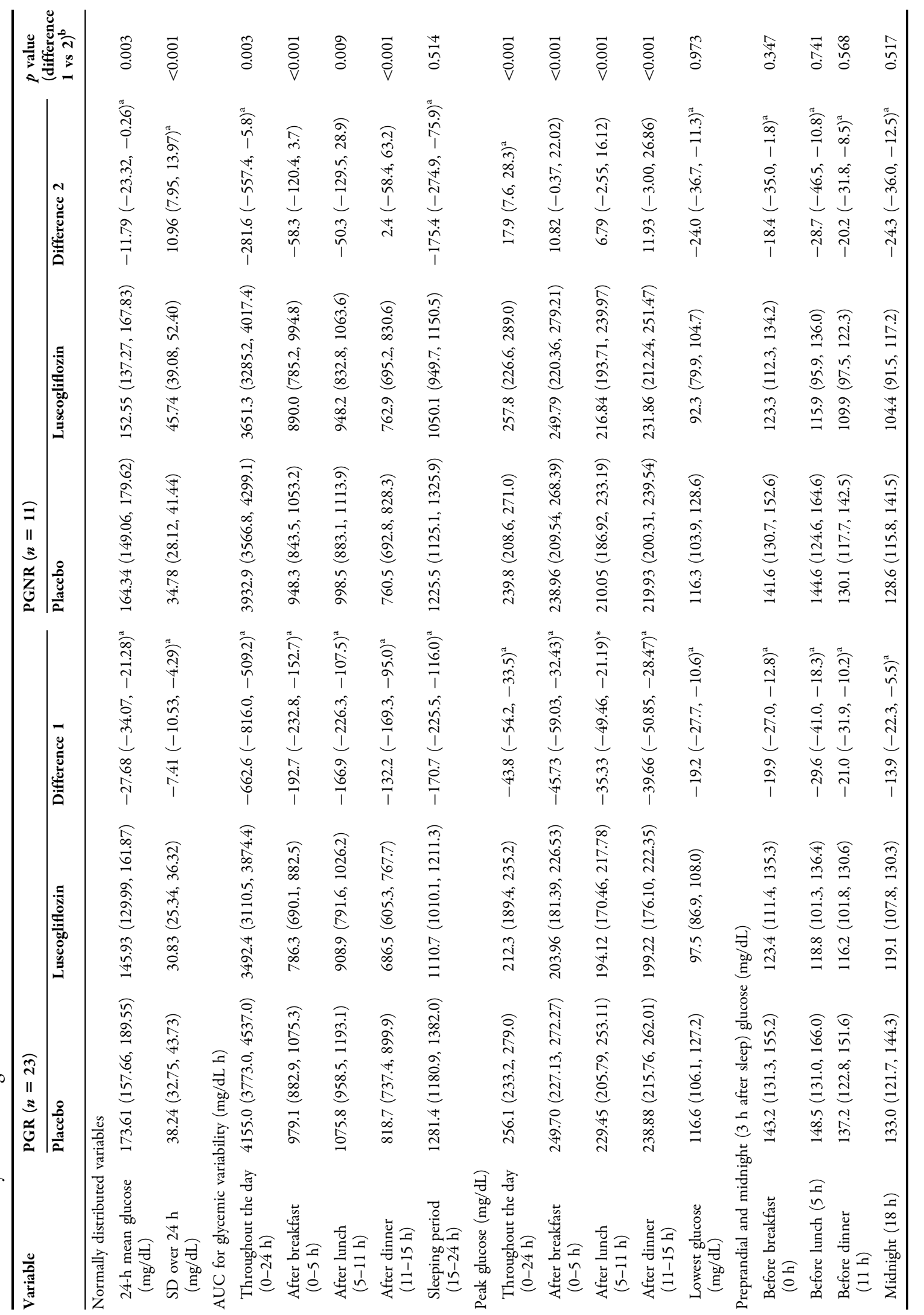




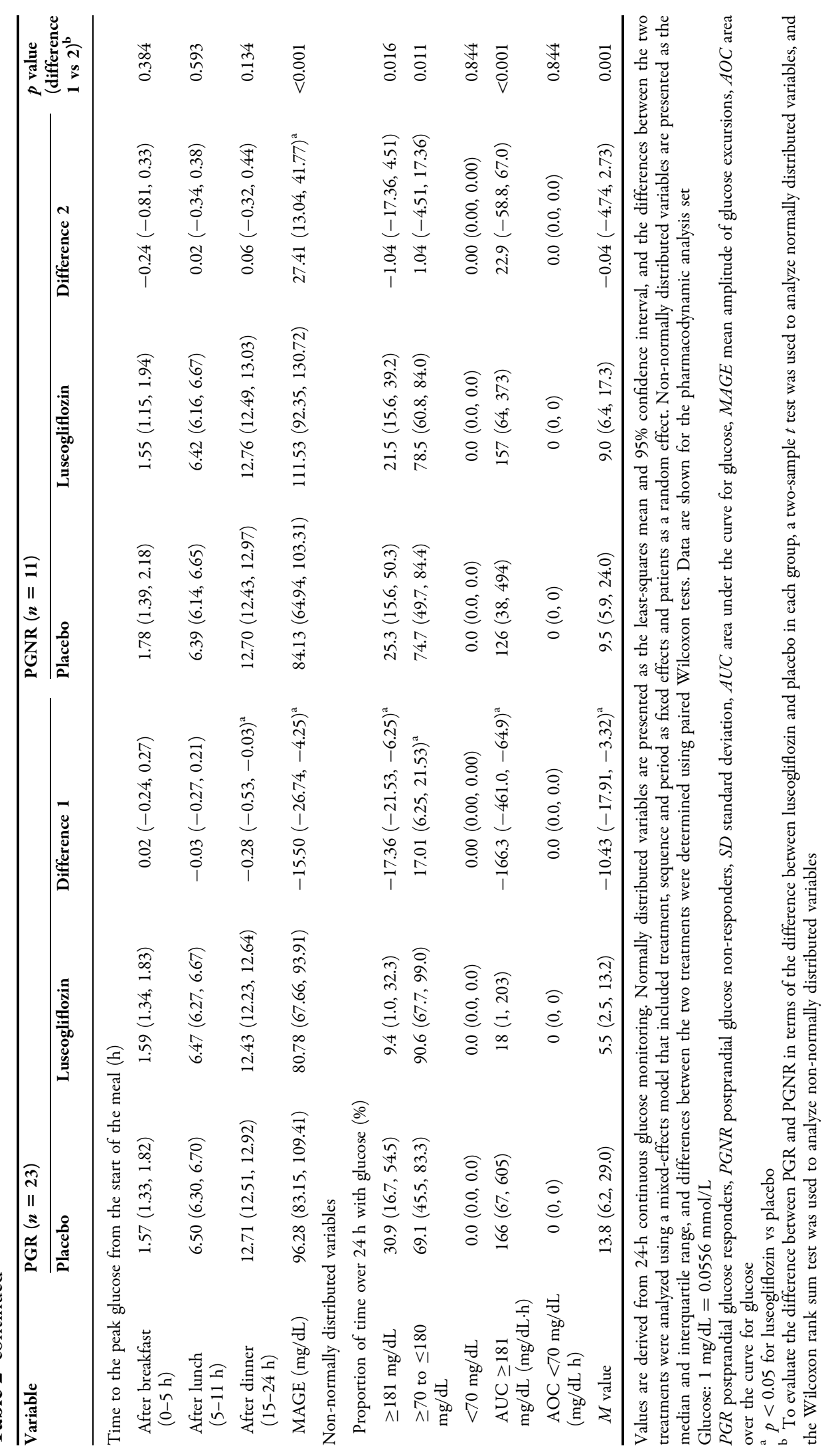



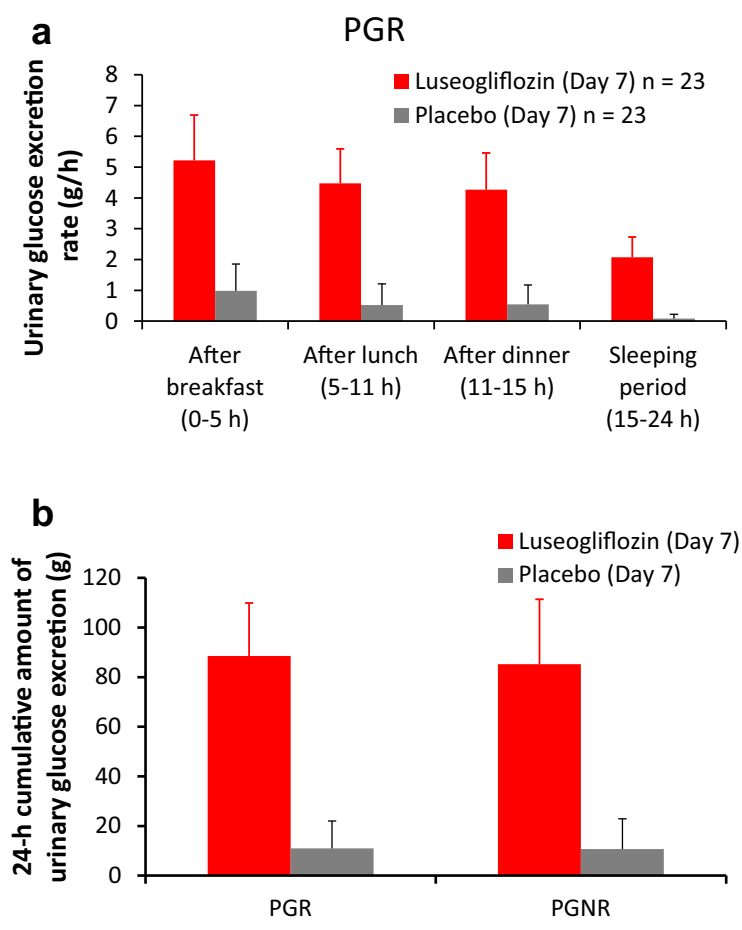

Fig. 2 Urinary glucose excretion. a Urinary glucose excretion rate and $\mathbf{b}$ 24-h cumulative amount of urinary glucose excretion. Values are presented as the mean +

at 0-24 h, 0-5 h, 5-11 h, 11-15 h, and 15-24 h on day 7 is shown in Fig. $2 \mathrm{~b}$ (0-24 h only) and Table 3. Luseogliflozin significantly increased the UGE rate and cumulative UGE by similar magnitudes in both groups (all $p<0.001$, Fig. 2; Table 3). The magnitude of the difference between luseogliflozin and placebo for the cumulative UGE was not significantly different between PGR and PGNR at each measurement period (Table 3).

The concentrations of serum insulin and plasma glucagon on day 7 with luseogliflozin and placebo in each group are shown in Fig. 3a and $\mathrm{b}$, respectively, and the area under the curve (AUC) and the maximum concentration of serum insulin and plasma glucagon at 0-24 h, 0-5 h, 5-11 h, 11-15 h, and 15-24 h on day 7 are shown in Table 3. Luseogliflozin decreased serum insulin throughout the 24-h

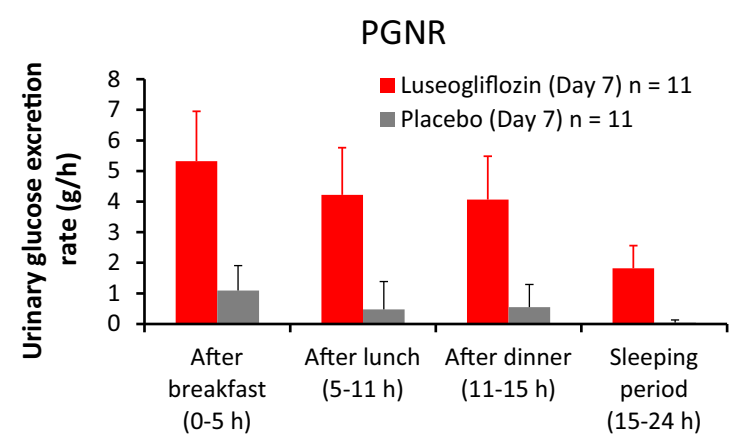

standard deviation. PGR postprandial glucose responders, $P G N R$ postprandial glucose non-responders

measurement period compared with placebo in both PGR and PGNR, and the AUC for serum insulin over $24 \mathrm{~h}$ was significantly decreased compared with placebo in both PGR and PGNR (both $p<0.05$, Table 3).

Luseogliflozin increased plasma glucagon throughout the 24-h measurement period compared with placebo in both PGR and PGNR (Fig. 3b), as well as the AUC for plasma glucagon over $24 \mathrm{~h}$ in the PGNR $(p<0.05$, Table 3).

The concentrations of serum acetoacetic acid and serum $\beta$-hydroxybutanoic acid on day 7 with luseogliflozin and placebo in the PGR and PGNR are shown in Fig. 4, and the AUC and the maximum concentration of serum acetoacetic acid and serum $\beta$-hydroxybutanoic acid at 0-24 h, 0-5 h, 5-11 h, 11-15 h, and 15-24 h on day 7 are shown in Table 3. Luseogliflozin 


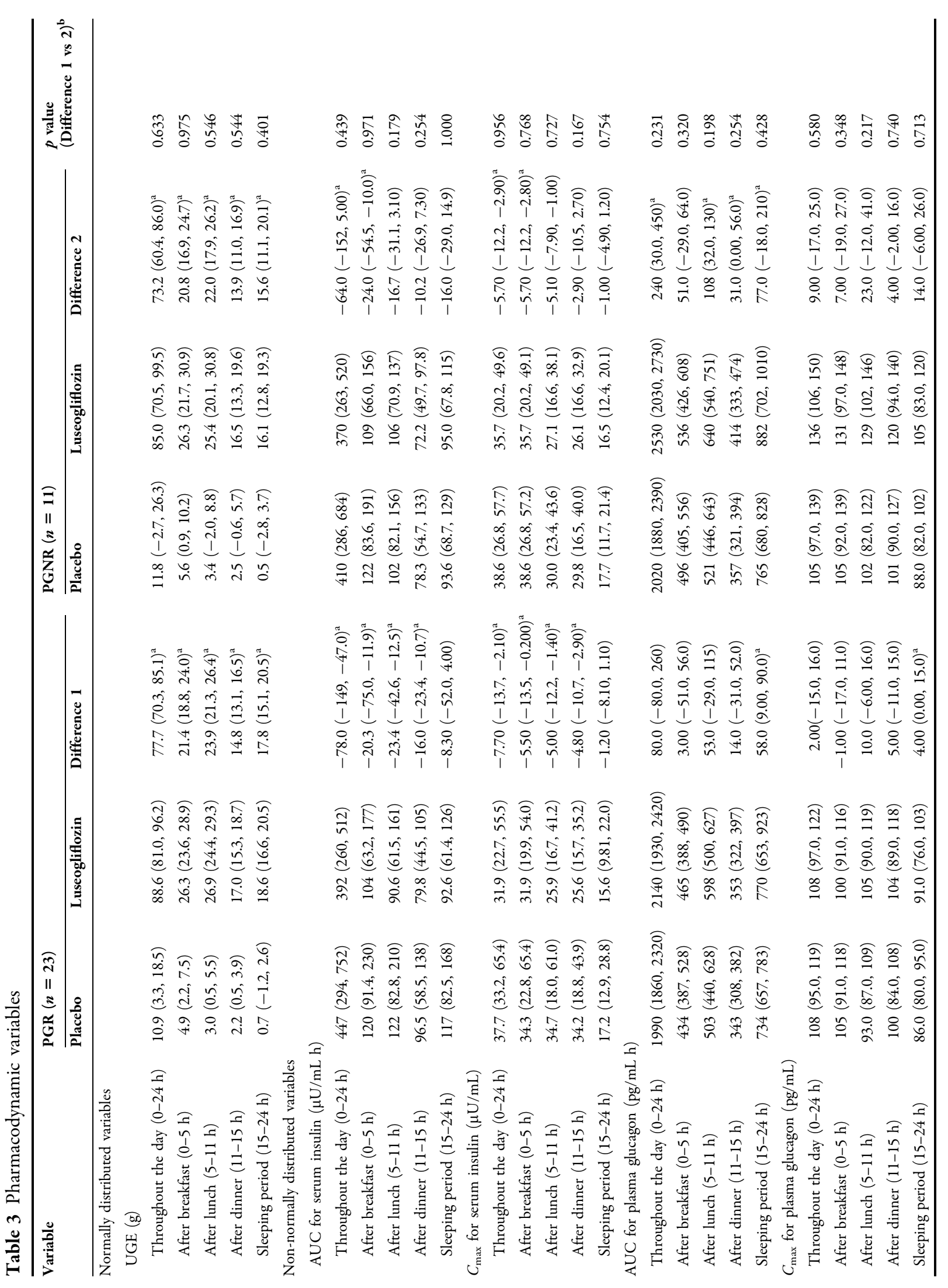




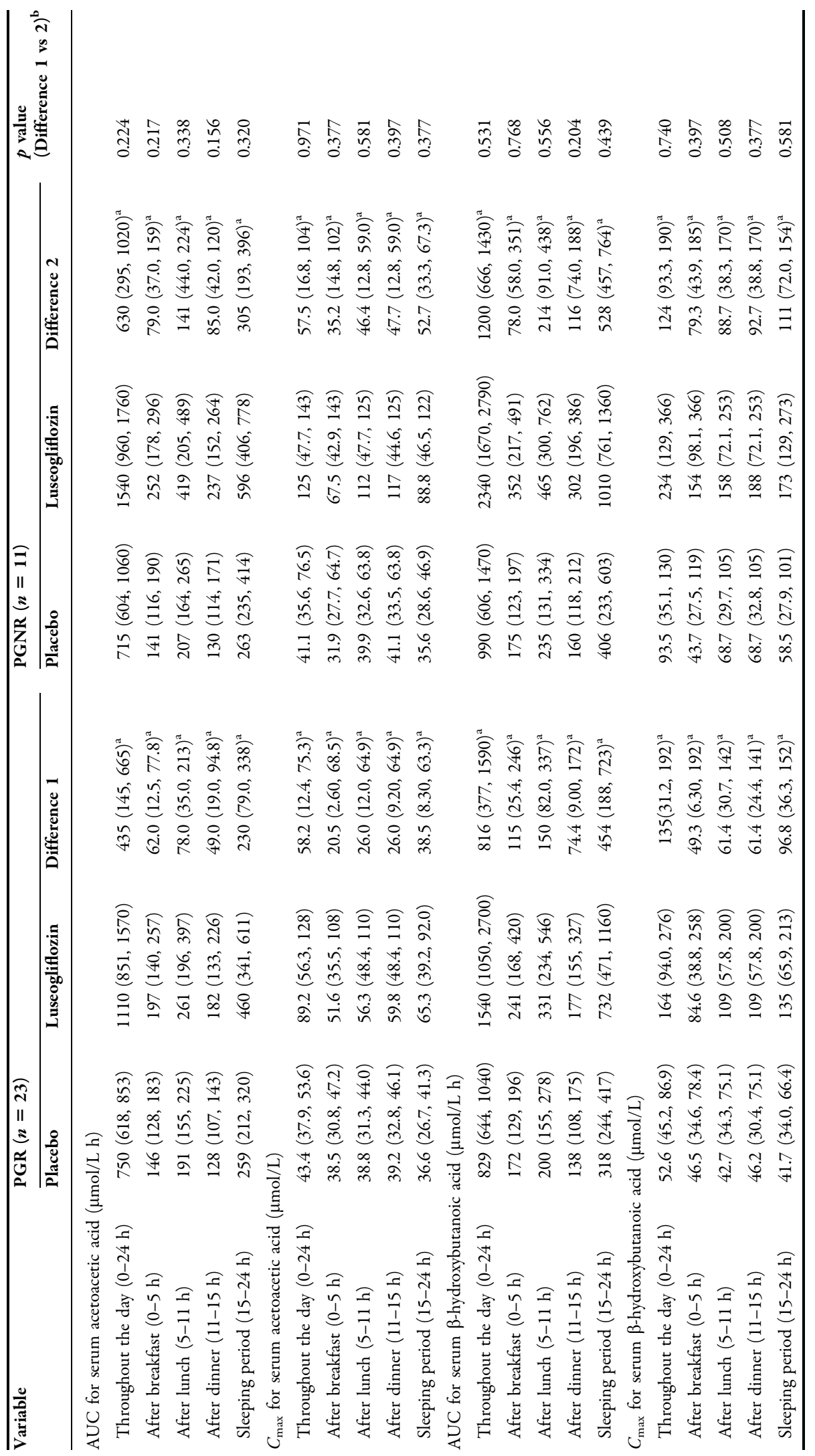




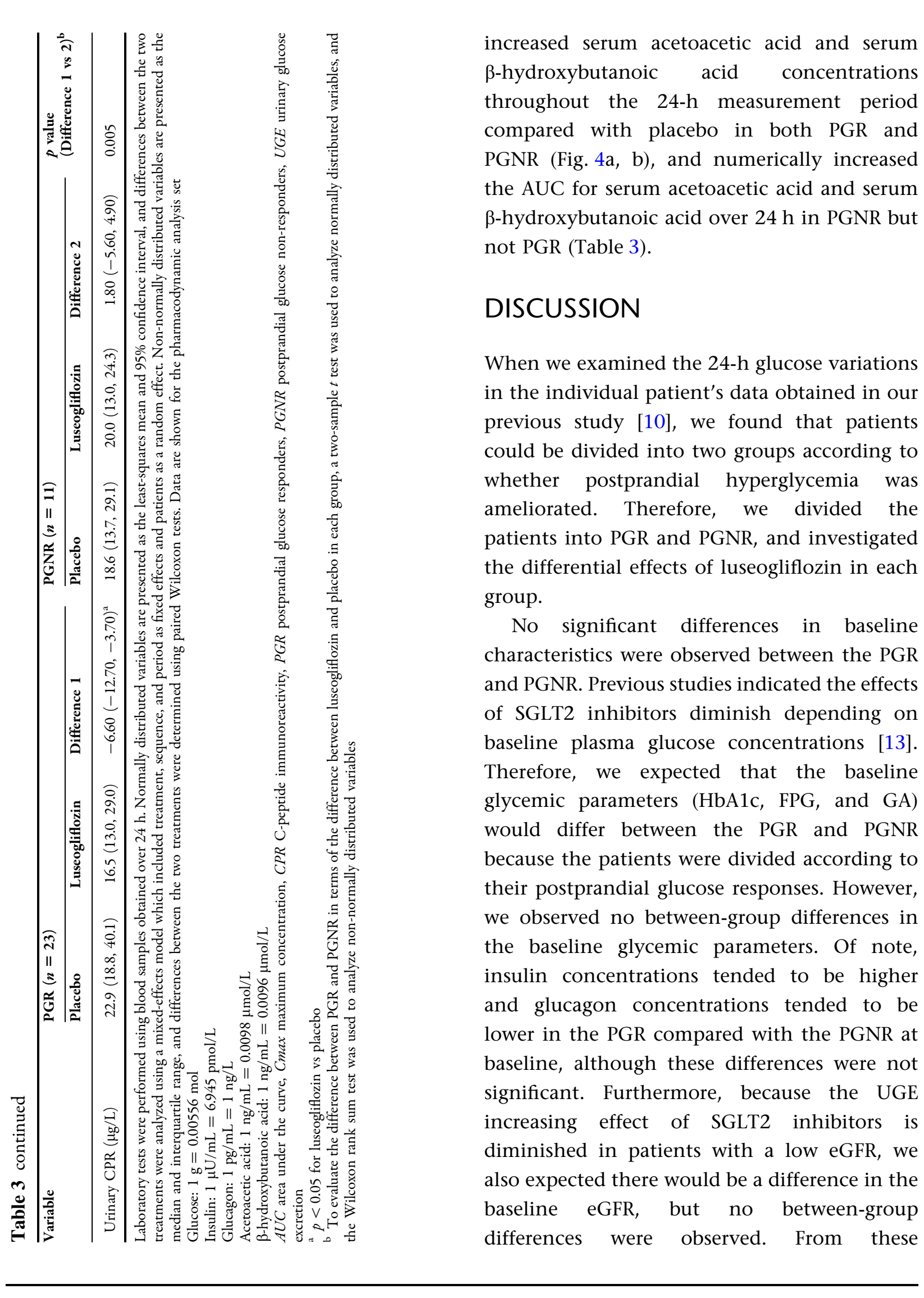



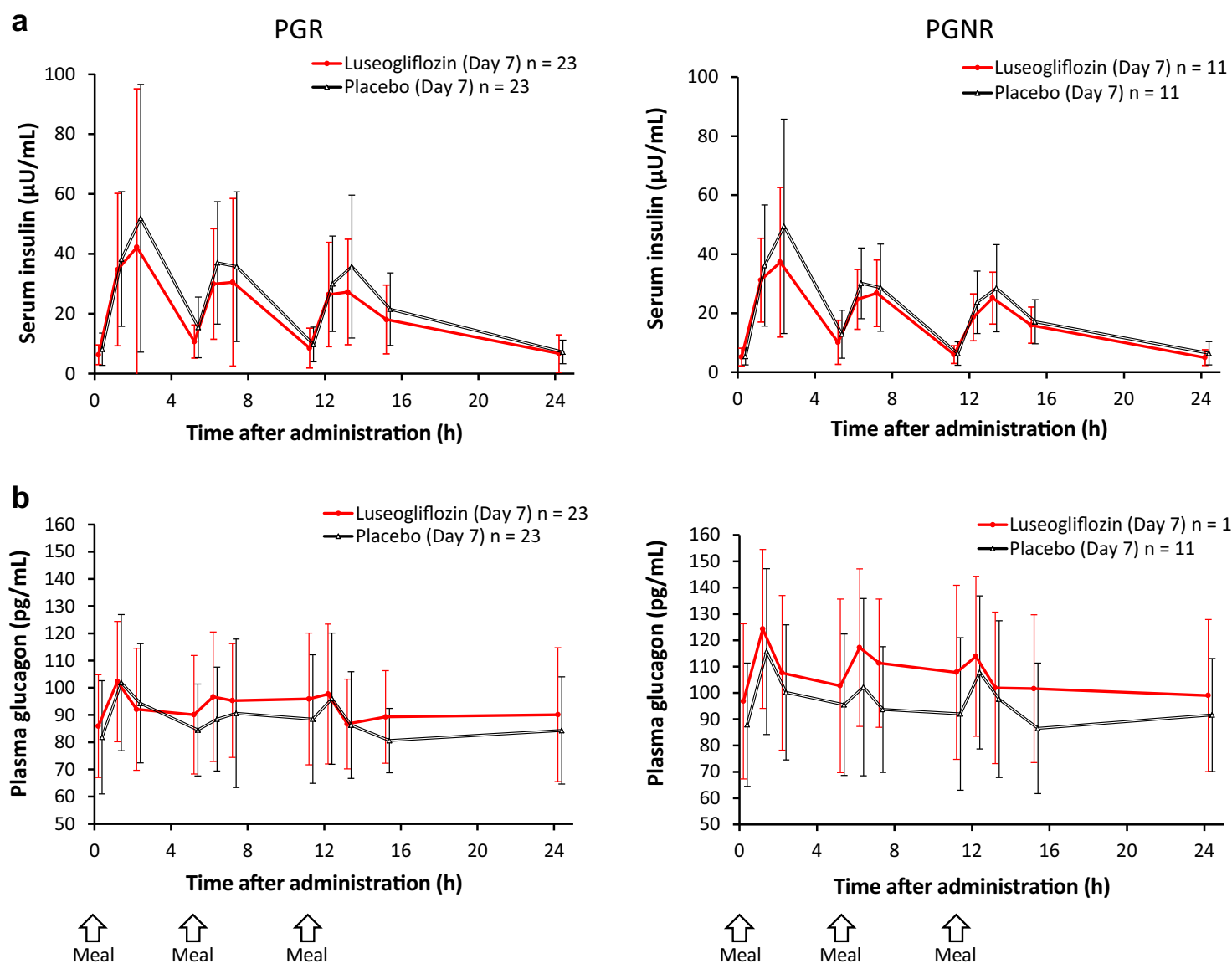

Fig. 3 Insulin and glucagon. a 24-h serum insulin concentration $(1 \mu \mathrm{U} / \mathrm{mL}=6.945 \mathrm{pmol} / \mathrm{L})$. b 24-h plasma glucagon concentration $(1 \mathrm{pg} / \mathrm{mL}=1 \mathrm{ng} / \mathrm{L})$. Values are

subanalyses, we could not identify which baseline characteristic determined the difference between the PGR and PGNR.

We previously investigated the hypoglycemic effect of an SGLT2 inhibitor using CGM [10, 11] and revealed a parallel downward shift involving the lowering of both fasting and postprandial glucose. However, in this subanalysis, luseogliflozin significantly diminished glucose fluctuations by lowering postprandial glucose by a greater magnitude than the reduction in fasting glucose in the PGR, which included over two-thirds of the study population. Given that SGLT2 inhibitors

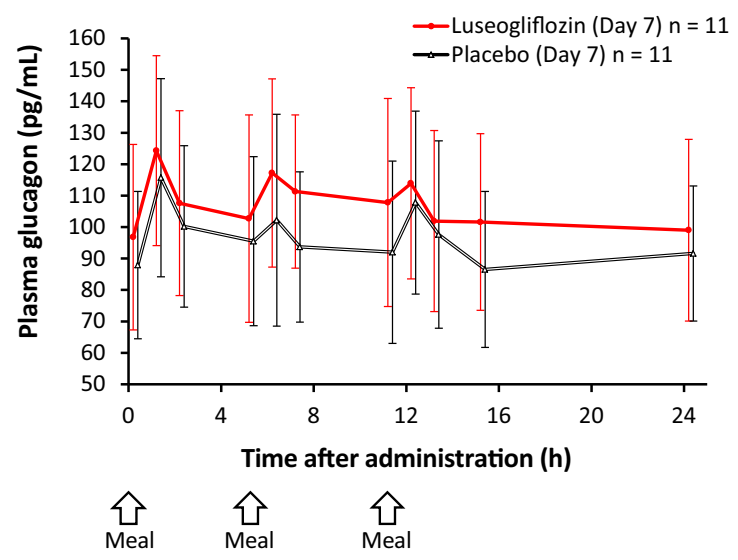

presented as the mean \pm standard deviation. $P G R$ postprandial glucose responders, $P G N R$ postprandial glucose non-responders

are reported to reduce glucose concentrations depending on baseline plasma glucose concentrations [13], it seems reasonable that greater hypoglycemic effects were observed at postprandial periods when glucose concentrations are higher. Based on the results of this subanalysis, we considered that luseogliflozin-treated patients might be stratified into two groups according to whether or not the postprandial glucose was ameliorated. When these two treatment effect patterns were analyzed in combination (mean value), we observed that the hypoglycemic effect of luseogliflozin followed a parallel downward shift. 

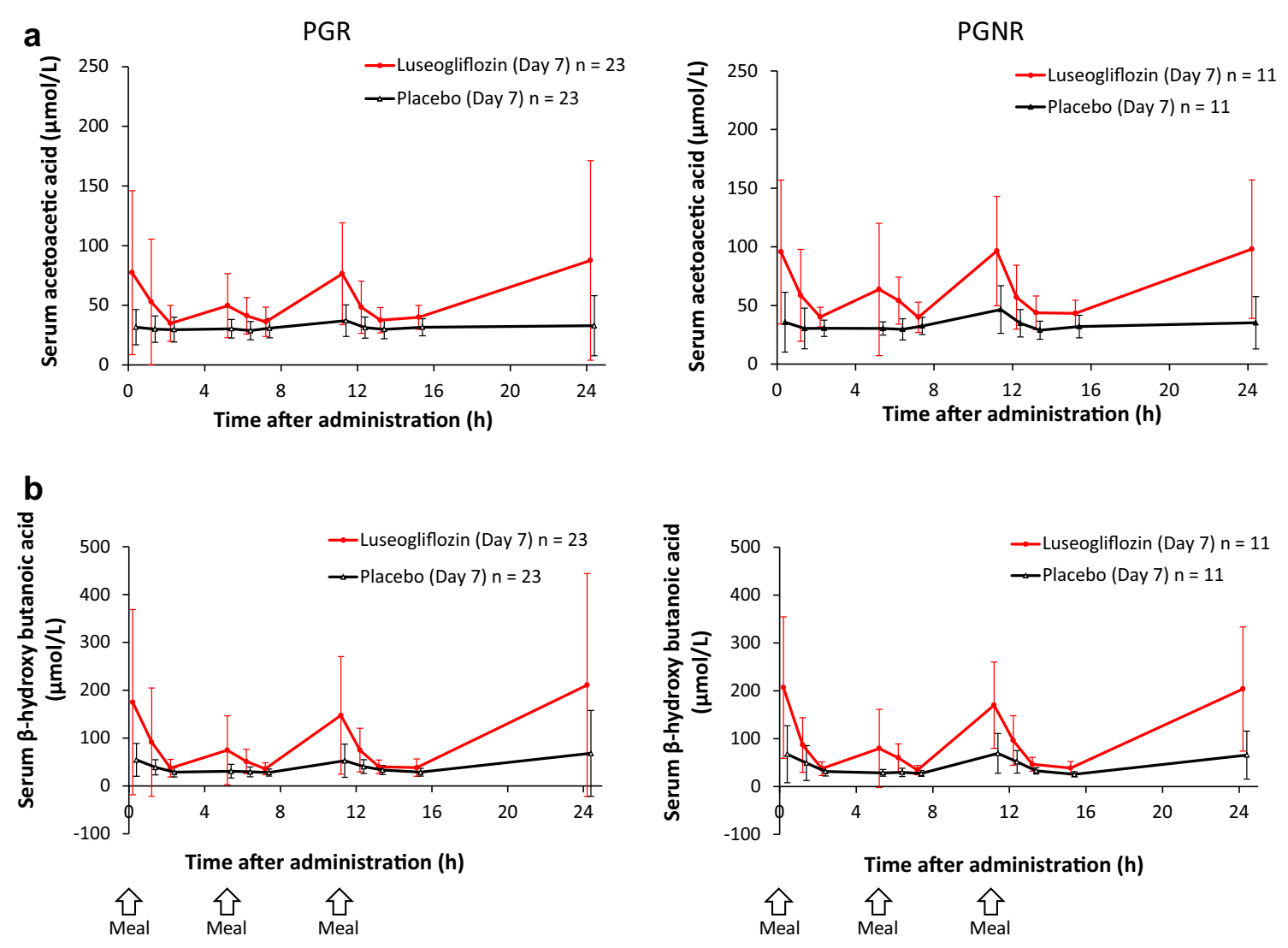

Fig. 4 Ketone bodies. a 24-h serum acetoacetic acid concentration $(1 \mathrm{ng} / \mathrm{mL}=0.0098 \mu \mathrm{mol} / \mathrm{L})$. b 24-h serum $\beta$-hydroxybutanoic acid $(1 \mathrm{ng} / \mathrm{mL}=0.0096 \mu \mathrm{mol} / \mathrm{L})$.

Interestingly, the magnitude of the increase in UGE, which is a direct pharmacological effect of luseogliflozin, was similar in the PGR and PGNR. Furthermore, the decrease in FPG in the PGNR was similar to that in the PGR. Because there was an apparent difference in the response to postprandial glucose levels between the PGR and PGNR, a difference in the increase in UGE and decrease in FPG was expected between these groups of patients. However, the increase in UGE and the decrease in FPG were similar in the PGR and PGNR. Therefore, we investigated other factors which might affect glucose concentrations.

The decrease of serum insulin secretion throughout the day by luseogliflozin was

Values are presented as the mean \pm standard deviation. $P G R$ postprandial glucose responders, $P G N R$ postprandial glucose non-responders

similar in each group and was consistent with previous studies [8, 14-16]. We expected the PGNR would require higher insulin concentrations compared with the PGR because the higher postprandial glucose concentrations in the PGNR might require more insulin secretion relative to the requirements in the PGR. However, the insulin concentrations decreased similarly in both PGR and PGNR.

Our subanalysis indicated that the difference in glucagon secretion after luseogliflozin administration might be a factor that determined the difference in response to postprandial hyperglycemia and might explain why the increase in postprandial hyperglycemia 
was not ameliorated in the PGNR, even though the increase in UGE and the decrease in FPG were similar in both the PGNR and PGR. SGLT2 inhibitors have been reported to increase plasma glucagon secretion and enhance endogenous glucose production [17]. In this subanalysis, glucagon secretion was increased in both the PGR and PGNR. Compared with the PGR, the increase in glucagon secretion in the PGNR was especially high during the postprandial periods, although this difference did not reach statistical significance (Fig. 3b). We consider that luseogliflozin increased glucagon secretion, enhanced gluconeogenesis, and induced acute glucose fluctuations that offset the glucose-lowering effect of luseogliflozin in postprandial periods in the PGNR. In addition, because luseogliflozin lowers plasma glucose concentrations by an indirect mechanism (increasing UGE), it seems feasible that luseogliflozin might not suppress sharp glucose fluctuations, while it could lower preprandial and midnight glucose concentrations not associated with acute glucose changes.

We propose a hypothesis whereby enhanced endogenous glucose production induced by an acute increase in glucagon secretion might mediate reductions in accumulated visceral fat, enhance glycogenolysis, and improve insulin resistance. Therefore, if luseogliflozin is used for a long period, postprandial hyperglycemia might be ameliorated even in PGNR. An acute increase in postprandial glucose might also be suppressed when luseogliflozin is used for long periods, even in PGNR, by exhausting accumulated glycogen concentrations. Glucose fluctuations might then be diminished in a similar manner in the PGR.

In this subanalysis, ketone bodies increased in both the PGR and PGNR; the increase was larger in the PGNR compared with the PGR although it did not reach statistical significance. It is thought that euglycemic diabetic ketoacidosis is induced by use of SGLT2 inhibitors and glucagon may also be involved [18]. Therefore, production of ketone bodies may be enhanced, particularly in the PGNR relative to the PGR. Meanwhile, no AEs related to ketone body increase (other than slight increases in laboratory parameters) occurred in either PGR or PGNR groups. Further investigation is needed to clarify the difference between the groups in the effects of luseogliflozin with respect to ketone body increase.

The present study had some limitations. It involved a subanalysis of our previous study [10], which was not powered for the analyses reported here. Although other factors might have affected the responses to postprandial hyperglycemia in patients treated with luseogliflozin, the interpretations of the present study are limited because of the inclusion/exclusion criteria of the original study. Additionally, luseogliflozin was only administered for 7 days, so the results might differ if the treatment period were longer. In the future, we plan to investigate the effects of long-term luseogliflozin treatment on 24-h glycemic variability.

\section{CONCLUSION}

The results of these post hoc subanalyses suggest that luseogliflozin diminishes glucose fluctuations in most patients by lowering postprandial glucose to a greater extent than its effects on fasting glucose. Luseogliflozin may also lower fasting glucose in patients whose postprandial glucose was not ameliorated despite an increase in UGE. Furthermore, the increase in glucagon secretion in patients whose postprandial glucose was not ameliorated might 
contribute to the differential hypoglycemic effects of luseogliflozin on postprandial glucose.

\section{ACKNOWLEDGMENTS}

Sponsorship, article processing charges, and the open access charge for this study were funded by Taisho Pharmaceutical Co., Ltd, Tokyo, Japan. All named authors meet the International Committee of Medical Journal Editors (ICMJE) criteria for authorship for this manuscript, take responsibility for the integrity of the work as a whole, and have given final approval to the version to be published. S.S. and Y.S. secured the research funding. Y.S. is the guarantor for this report. All authors had full access to all of the data in this study and take complete responsibility for the integrity of the data and accuracy of the data analysis. Editorial assistance in the preparation of this manuscript was provided by Nicholas D. Smith, Ph.D., and Michelle Belanger, MD, of Edanz Group Ltd., which was funded by Taisho Pharmaceutical Co., Ltd.

Disclosures. R. Nishimura has received consultancy fees or lecture fees from Abbott Diabetes Care, Inc., Astellas Pharma US, Inc., AstraZeneca Pharmaceuticals LP, Boehringer Ingelheim Pharmaceuticals, Inc., Eli Lilly Japan K.K., Kissei Pharmaceutical Co., Ltd., Novartis Corporation, Novo Nordisk Pharma Ltd., Sanofi K.K., Taisho Pharmaceutical Co., Ltd., Taisho Toyama Pharmaceutical Co., Ltd., and Takeda Pharmaceutical Company Limited. H. Omiya, H. Watase, K. Nozaki, S. Sakai and Y. Samukawa are employees of Taisho Pharmaceutical Co., Ltd., which is developing luseogliflozin.

Open Access. This article is distributed under the terms of the Creative Commons
Attribution-NonCommercial 4.0 International License (http://creativecommons.org/licenses/ by-nc/4.0/), which permits any noncommercial use, distribution, and reproduction in any medium, provided you give appropriate credit to the original author(s) and the source, provide a link to the Creative Commons license, and indicate if changes were made.

\section{Compliance with Ethics Guidelines All} procedures followed were in accordance with the ethical standards of the responsible committee on human experimentation (institutional and national) and with the Helsinki Declaration of 1964, as revised in 2013. Informed consent was obtained from all patients for being included in the study.

\section{REFERENCES}

1. American Diabetes Association. Standards of medical care in diabetes-2016. Diabetes Care. 2016;39:S1-112.

2. Bays H. Sodium glucose co-transporter type 2 (SGLT2) inhibitors: targeting the kidney to improve glycemic control in diabetes mellitus. Diabetes Ther. 2013;4:195-220.

3. Kurosaki E, Ogasawara H. Ipragliflozin and other sodium-glucose cotransporter-2 (SGLT2) inhibitors in the treatment of type 2 diabetes: preclinical and clinical data. Pharmacol Ther. 2013;139:51-9.

4. Tahrani AA, Barnett AH, Bailey CJ. SGLT inhibitors in management of diabetes. Lancet Diabetes Endocrinol. 2013;1:140-51.

5. Markham A, Elkinson S. Luseogliflozin: first global approval. Drugs. 2014;74:945-50.

6. Kim GW, Chung SH. Clinical implication of SGLT2 inhibitors in type 2 diabetes. Arch Pharm Res. 2014;37:957-66.

7. Ferrannini E, Solini A. SGLT2 inhibition in diabetes mellitus: rationale and clinical prospects. Nat Rev Endocrinol. 2012;8:495-502.

8. Jurczak MJ, Lee HY, Birkenfeld AL, et al. SGLT2 deletion improves glucose homeostasis and 
preserves pancreatic beta-cell function. Diabetes. 2011;60:890-8.

9. Seino Y, Sasaki T, Fukatsu A, Ubukata M, Sakai S, Samukawa Y. Efficacy and safety of luseogliflozin as monotherapy in Japanese patients with type 2 diabetes mellitus: a randomized, double-blind, placebo-controlled, phase 3 study. Curr Med Res Opin. 2014;30:1245-55.

10. Nishimura R, Osonoi T, Kanada S, et al. Effects of luseogliflozin, a sodium-glucose co-transporter 2 inhibitor, on 24-hour glucose variability assessed by continuous glucose monitoring in Japanese patients with type 2 diabetes mellitus: a randomized, double-blind, placebo-controlled, crossover study. Diabetes Obes Metab. 2015;17:800-4.

11. Nishimura R, Omiya H, Sugio K, Ubukata M, Sakai $\mathrm{S}$, Samukawa Y. The sodium-glucose cotransporter 2 inhibitor luseogliflozin improves glycaemic control assessed by continuous glucose monitoring even on a low-carbohydrate diet. Diabetes Obes Metab. 2015;. doi:10.1111/dom.12611.

12. Nishimura R, Tanaka Y, Koiwai K, et al. Effect of empagliflozin monotherapy on postprandial glucose and 24-hour glucose variability in Japanese patients with type 2 diabetes mellitus: a randomized, double-blind, placebo-controlled, 4-week study. Cardiovasc Diabetol. 2015;14:11.

13. Yamada $\mathrm{K}$, Nakayama $\mathrm{H}$, Yoshinobu S, et al. Effects of a sodium glucose co-transporter 2 selective inhibitor, ipragliflozin, on the diurnal profile of plasma glucose in patients with type 2 diabetes: a study using continuous glucose monitoring. J Diabetes Investig. 2015;6:699-707.

14. Seino Y, Sasaki T, Fukatsu A, Sakai S, Samukawa Y. Efficacy and safety of luseogliflozin monotherapy in Japanese patients with type 2 diabetes mellitus: a 12-week, randomized, placebo-controlled, phase II study. Curr Med Res Opin. 2014;30:1219-30.

15. Seino Y, Sasaki T, Fukatsu A, Ubukata M, Sakai S, Samukawa Y. Dose-finding study of luseogliflozin in Japanese patients with type 2 diabetes mellitus: a 12-week, randomized, double-blind, placebo-controlled, phase II study. Curr Med Res Opin. 2014;30:1231-44.

16. Sasaki T, Seino Y, Fukatsu A, Ubukata M, Sakai S, Samukawa Y. Pharmacokinetics, pharmacodynamics, and safety of luseogliflozin in Japanese patients with type 2 diabetes mellitus: a randomized, single-blind, placebo-controlled trial. Adv Ther. 2015;32:319-40.

17. Bonner C, Kerr-Conte J, Gmyr V, et al. Inhibition of the glucose transporter SGLT2 with dapagliflozin in pancreatic alpha cells triggers glucagon secretion. Nat Med. 2015;21:512-7.

18. Ogawa W, Sakaguchi K. Euglycemic diabetic ketoacidosis induced by SGLT2 inhibitors: possible mechanism and contributing factors. J Diabetes Investig. 2016;7:135-8. 